\title{
Neuromonitoring in intensive care: a new brain tissue probe for combined monitoring of intracranial pressure (ICP) cerebral blood flow (CBF) and oxygenation
}

\author{
Keller, E ; Fröhlich, J ; Muroi, C ; Sikorski, C ; Muser, M
}

\begin{abstract}
BACKGROUND: the benefits of monitoring cerebral blood flow (CBF) in stroke patients are apparent. New techniques combining near infrared spectroscopy (NIRS) and indocyanine green (ICG) dye dilution to estimate cerebral hemodynamics are available. However, with transcutaneous NIRS and optodes applied over the skin, the signal is contaminated by extracerebral tissues. The objective is to develop a new brain tissue probe for combined monitoring of intracranial pressure (ICP), CBF and cerebral blood volume (CBV). METHODS: conventional intraparenchymal probes for ICP monitoring are supplied with optical fibers. The light is coupled into the brain tissue and collected after absorption and scattering with a light detector. Venous injections of $0.2 \mathrm{mg} / \mathrm{kgbw}$ ICG are performed. The mean transit time of ICG (mttICG), CBF and CBV are calculated. RESULTS: with a prototype of the probe in a first patient with subarachnoid hemorrhage 6 pairs of repetitive measurements were performed. Mean values were for mttICG $5.6 \pm 0.2 \mathrm{~s}$, CBF $22.3 \pm 2.8 \mathrm{ml} / 100 \mathrm{~g} / \mathrm{min}$ and CBV $2.1 \pm 0.3 \mathrm{ml} / 100 \mathrm{~g}$. CONCLUSIONS: NIR spectroscopy allows the synchronous determination of multiple parameters with one single device. By measurements in parallel with the NeMo Probe and NIRS optodes placed over the skin, new algorithms can be developed to subtract the extracerebral contamination from the NIRS signal.
\end{abstract}

DOI: https://doi.org/10.1007/978-3-7091-0356-2_39

Posted at the Zurich Open Repository and Archive, University of Zurich ZORA URL: https://doi.org/10.5167/uzh-45190

Book Section

Originally published at:

Keller, E; Fröhlich, J; Muroi, C; Sikorski, C; Muser, M (2011). Neuromonitoring in intensive care: a new brain tissue probe for combined monitoring of intracranial pressure (ICP) cerebral blood flow (CBF) and oxygenation. In: Feng, H; Mao, Y; Zhang, J H. Early Brain Injury or Cerebral Vasospasm Volume 2: Clinical Management. Wien: Springer, 217-220.

DOI: https://doi.org/10.1007/978-3-7091-0356-2_39 
Neuromonitoring in Intensive Care: A New Brain Tissue Probe for Combined

Monitoring of Intracranial Pressure (ICP) Cerebral Blood Flow (CBF) and Oxygenation

E. Keller ${ }^{1}$, J. Froehlich $^{2}$, C. Muroi ${ }^{1}$, C. Sikorski ${ }^{1}$, and M. Muser ${ }^{3}$

1 Neurocritical Care Unit, Department of Neurosurgery, University Hospital Zurich, Zurich, Switzerland

2 Laboratory for Electromagnetic Fields \& Microwave Electronics, ETH Zuerich, Switzerland

3 NeMoDevices, Zuerich, Switzerland

Address of correspondence:

Emanuela Keller, MD

Neurointensive Care Unit

Dept. of Neurosurgery

University Hospital

Frauenklinikstrasse 10

CH-8091 Zuerich

Switzerland

E-mail: emanuela.keller@usz.ch

Tel.: +4144255 5671

Fax: +41 442554672 


\section{Summary:}

Background: The benefits of monitoring cerebral blood flow (CBF) in stroke patients are apparent. New techniques combining near infrared spectroscopy (NIRS) and indocyanine green (ICG) dye dilution to estimate cerebral hemodynamics are available. However, with transcutaneous NIRS and optodes applied over the skin, the signal is contaminated by extracerebral tissues. The objective is to develop a new brain tissue probe for combined monitoring of intracranial pressure (ICP), CBF and cerebral blood volume (CBV).

Methods: Conventional intraparenchymal probes for ICP monitoring are supplied with optical fibers. The light is coupled into the brain tissue and collected after absorption and scattering with a light detector. Venous injections of $0.2 \mathrm{mg} / \mathrm{kgbw}$ ICG are performed. The mean transit time of ICG (mttICG), CBF and CBV are calculated.

Results: With a prototype of the probe in a first patient with subarachnoid hemorrhage 6 pairs of repetitive measurements were performed. Mean values were for mttICG 5.6 $\pm 0.2 \mathrm{sec}$, $\mathrm{CBF} 22.3 \pm 2.8 \mathrm{ml} / 100 \mathrm{~g} / \mathrm{min}$ and $\mathrm{CBV} 2.1 \pm 0.3 \mathrm{ml} / 100 \mathrm{~g}$

Conclusions: NIR spectroscopy allows the synchronous determination of multiple parameters with one single device. By measurements in parallel with the NeMo Probe and NIRS optodes placed over the skin, new algorithms can be developed to subtract the extracerebral contamination from the NIRS signal.

Keywords: Subarachnoid haemorrhage, traumatic brain injury, cerebral blood flow, brain tissue probe, intracranial pressure 


\section{Introduction:}

The benefits of monitoring cerebral blood flow (CBF) in patients with subarachnoid hemorrhage, severe hemispheric stroke and head injury are apparent. Numerous techniques have been developed to estimate cerebral hemodynamics $(1,5,10,11,14,19)$. However, each method has its own advantages and drawbacks. To date a suitable method for bedside CBF measurement, able to detect smaller areas of ischemia and easy to perform at the bedside is still lacking.

Optical methodologies may be the ideal instrument to monitor disease-related secondary brain injuries in instable patients in the environment of intensive care, emergency care and surgical units. New techniques combining near infrared spectroscopy (NIRS) and indocyanine green (ICG) dye dilution to estimate cerebral hemodynamics are available $(3,7,8,13,15)$. However, transcutaneous NIRS with optodes applied over the skin is controversially discussed, because the NIRS signal is contaminated by extracerebral tissues (skin, skull, cerebrospinal fluid layer) $(4,6,17)$. To obtain measurement values directly from the brain tissue a conventional probe for intracranial pressure (ICP) monitoring can be supplied with optical fibers for NIRS. For patients with severe brain injuries and stroke, where ICP probes are installed anyway because of brain oedema and intracranial hypertension, a new combined NIRS ICP probe may offer enhanced modality without an additional surgical intervention.

The objective is to develop a new intraparenchymal probe for combined monitoring of ICP, brain temperature, CBF and cerebral blood volume (CBV) with NIRS and ICG dye dilution. 


\section{Methods:}

The study was approved by the Ethics Committee of the University of Zurich. Measurements were performed in a patient with severe subarachnoid hemorrhage Hunt and Hess grade 5, Fisher 4 with ruptured aneurysm of the anterior communicating artery. Because of occlusive hydrocephalus a ventricular catheter (Bactiseal ${ }^{\circledR}$, Codman, Johnson \& Johnson, USA) was inserted to drain cerebrospinal fluid. After coiling the ruptured aneurysm, the patient developed brain edema and ICP monitoring with an intraparenchymal probe was needed. A prototype of the new NIRS ICP probe (NeMo Probe ${ }^{\circledR}$, NeMoDevices AG, Switzerland) was inserted through a support bolt (Raumedic $®$, Germany) from a burr hole by an experienced neurosurgeon in the ICU (figure 1). The probe was placed into the brain tissue, $2 \mathrm{~cm}$ deep from the dura. The correct positioning of the probe was confirmed with computed tomography (CT) (figure 2).

For NIRS, conventional intraparenchymal probes for ICP monitoring (diameter 6 French) were supplied with optical fibers (NeMo Probe ${ }^{\circledR}$, NeMoDevices, Switzerland). The light is coupled out into the brain tissue via a metalized microprism and collected after absorption and scattering with a light detector. A thermistor and an ICP sensor (microminiature strain gauge pressure sensor) were included at the tip of the probe. Before implantation the ICP sensors were extensively tested over 8 days and showed no significant measurable drift. A NIRS apparatus, specifically constructed for the measurement mode (NeMo System®, NeMoDevices, Zuerich, Switzerland) include the light sources, the hardware for data collection and the software to analyze the NIRS data (NeMo View®) (figure 3). Regular measurements were performed daily and repeated after 15 mins under stable clinical conditions (unchanged ICP, mean arterial pressure, $\mathrm{paCO}_{2}$ ). Central venous injections of $0.2 \mathrm{mg} / \mathrm{kgbw}$ ICG were performed, followed by the injection of $10 \mathrm{ml}$ Glucose $5 \%$ flush. The ICG concentrations were calculated based on the changes in OD. Regional values for the 
mean transit time of ICG (mttICG), CBF and CBV were calculated according to published algorithms (8).

Statistical analysis: Standard deviation and coefficient of variation were calculated for repeated measurements. ICP values were compared using the correlation coefficient and paired t-test.

\section{Results:}

In a first patient with subarachnoid hemorrhage, with two prototypes of the new brain tissue probe 16 measurements with ICG injections were performed on 4 consecutive days. No complications associated with the measurement technique occurred. The user-acceptance by neurosurgeons and ICU staff was as high as for conventional brain tissue probes for ICP monitoring. Day 3, the first probe had to be changed because of decreased signal to noise ratio. Day 5, before an additional measurement could be performed, the probe was pulled out by the nurse accidentally. In 12 single, 6 pairs of measurements, data analysis was successful. Mean values were for mttICG $5.6 \pm 0.2 \mathrm{sec}, \mathrm{CBF} 22.3 \pm 2.8 \mathrm{ml} / 100 \mathrm{~g} / \mathrm{min}$ and CBV $2.1 \pm 0.3$ $\mathrm{ml} / 100 \mathrm{~g}$. The coefficients of variation for repetitive measurements were for mttICG 0.046, CBV 0.18 and CBF 0.14. 12134 ICP values obtained with the NeMo Probe (ICP $\mathrm{NeMo}_{\mathrm{N}}$ ) were compared with ICP values measured with a conventional ventricular drainage and an external tranducer $\left(\mathrm{ICP}_{\mathrm{VD}}\right)$. The correlation coefficient between $\mathrm{ICP}_{\mathrm{NeMo}}$ and $\mathrm{ICP}_{\mathrm{VD}}$ was 0.7219 (95\% CI 0.7132 to $0.7303 ; \mathrm{p}<0.0001)$.

\section{Discussion:}

Originally, as scientific prototypes, the optical fibers for combined NIRS and ICP monitoring were first integrated into a conventional subdural probe for ICP-monitoring and tested in two patients (9). Still, in clinical practice the use of ICP probes constructed as 
subdural types is not common yet, as intraparenchymal probes are preferred due to their higher measuring accuracy and their smaller diameter which makes their application less invasive $(2,16)$. Therefore, the industrialized prototypes are now being produced as probes to be inserted directly into the brain tissue.

Two prototypes of the new intraparenchymal NIRS ICP probe could be tested in a first patient and values for mttICG, $\mathrm{CBF}$ and $\mathrm{CBV}$ could be determined. The measured values obtained with the NIRS ICP probe with a mean CBF of $22.3 \pm 2.8 \mathrm{ml} / 100 \mathrm{~g} / \mathrm{min}$ and a mean $\mathrm{CBV}$ of $2.1 \pm 0.3 \mathrm{ml} / 100 \mathrm{~g}$ correspond to normal values obtained with $\mathrm{H}_{2}{ }^{15} \mathrm{O}$ positron emission tomography $(\mathrm{PET})(22.2 \pm 4.9 \mathrm{ml} / 100 \mathrm{~g} / \mathrm{min}$ for $\mathrm{CBF}$ and $2.7 \pm 0.56 \mathrm{ml} / 100 \mathrm{~g}$ for CBV) or with dynamic susceptibility contrast magnetic resonance imaging (MRI) $(23 \pm 14$ $\mathrm{ml} / 100 \mathrm{~g} / \mathrm{min}$ for $\mathrm{CBF}$ and $1.3 \pm 0.4 \mathrm{ml} / 100 \mathrm{~g}$ for $\mathrm{CBV})$ in the white matter $(12,18)$. To test the accuracy of the measurement method, in a very first step its reproducibility was examined with repeated measurements under unchanged clinical conditions. The standard deviations and coefficients of variation are clinically well acceptable.

For patients with severe brain injuries and stroke, where ICP probes are anyway installed because of brain oedema and intracranial hypertension, the NeMo Probe offers enhanced modality modes (ICP, brain temperature, mttICG, CBF, CBV, changes of oxygenated, desoxygenated and total hemoglobin concentrations) without an additional surgical intervention. The new multimodal monitoring system provides information of cerebral hemodynamics, oxygenation and metabolism synchronously within minutes at the bedside and may allow optimising and individualising new neuroprotective therapies for every single brain injured patient.

Like other methods applying brain tissue probes, as microdialysis or brain tissue oxygen tension pressure $\left(\mathrm{PbtO}_{2}\right)$ monitoring, the major restriction of the NeMo System applying the NIRS ICP probe is that it is a regional measurement method, giving relevant results only if 
the probe is inserted into the area of interest. Cerebral vasospasm leading to focal ischemic events after subarachnoid hemorrhage may occur in different and multiple vascular territories, presumably not being observed by the NeMo Probe. Ongoing theoretical examinations applying different model simulations will clarify the extent of the measurement volume of the NeMo Probe, which depends not only on the distance between the light emitter and detector, but on different light scattering properties under specific pathophysiological conditions in the brain tissue.

The advantages of patches with NIRS optodes applied over the skin, is that they are strictly non-invasive. Several vascular territories, therefore, can be monitored over both hemispheres symmetrically. Furthermore, measurements with the NeMo Probe and optode patches over the skin in parallel, will allow for the development of algorithms to determine and subtract extracerebral contamination. In less severely ill patients, not requiring an ICP monitoring probe and in patients treated with full anticoagulation (e.g. during cardiopulmonary bypass surgery) the non invasive approach with optode patches attached over the skin and optimized algorithms may be the first choice.

In conclusion: The new combined NIRS ICP probe allows the determination of multiple parameters synchronously with one single device. By measurements in parallel with the NeMo Probe and NIRS optodes placed over the skin, new algorithms will be developed to determine the contribution from extracerebral tissues within the cumulative signal obtained by transcranial NIRS. 


\section{Figure legends:}

Figure 1: Dark: NeMo Probe inserted through a support bolt from a burr hole; bright: ventricular drainage

Figure 2: Computed tomography (CT) scans showing the NeMo Probe and the ventricular drainage (coronar section, bone window)

Figure 3: NeMo System including light sources, data collection unit and software for data analysis

\section{Source of Funding:}

The study was supported by NeMoDevices AG, Zuerich, Switzerland

\section{Disclosures:}

E. Keller, J. Froehlich and M. Muser have a financial interest as founder, resp. member of staff of NeMoDevices AG.

\section{References:}

1. Aaslid R, Huber P, Nornes H (1984) Evaluation of cerebrovascular spasm with transcranial doppler ultrasound. J Neurosurg 60: 37-41

2. Bhatja A, Gupta AK (2007) Neuromonitoring in the intensive care unit.I. Intracranial pressure and cerebral blood flow monitoring. Intensive Care Med 33:1263-1271 
3. Colacino JM, Grubb B, Jobsis FF (1981) Infra-red technique for cerebral blood flow: comparison with xenon 133 clearance. Neurol Res 3:17-31

4. Germon TJ, Evans PE, Barnett NJ, Lewis TT, Wall P, Nelson RJ (1997) Changes in tissue oxyhemoglobin concentration measured using multichannel near infrared spectroscopy during internal carotid angiography. J Neurol Neurosurg Psychiat 63:660-664

5. Heiss WD, Graf R, Löttgen J, Ohta K, Fujita T, Wagner R, Grond M, Weinhard K (1997) Repeat positron emission tomographic studies in transient middle cerebral artery occlusion in cats: residual perfusion and efficiacy of postischemic reperfusion. J Cerebr Blood Flow Metabol 17:388-400

6. Hongo K, Kobayashi S, Okudera H, Hokama M, Nakagawa F (1995) Noninvasive cerebral optical spectroscopy: Depth-resolved measurements of cerebral hemodynamics using indocyanine green. Neurol Res 17:89-93

7. Hopton P, Walch TS, Lee A (1999) Measurement of cerebral blood volume using nearinfrared spectroscopy and indocyanine green elimination. J Appl Physiol 87:1981-1987

8. Keller E, Nadler A, Alkhadi H, Kollias S, Yonekawa Y, Niederer P (2003) Non invasive measurement of regional cerebral blood flow and regional cerebral blood volume by near infrared spectroscopy and indocyanine green dye dilution. Neuroimage 20:828-839

9. Keller E, Nadler A, Niederer P, Yonekawa Y, Imhof HG (2002) A new subdural probe for combined intracranial pressure (ICP) and cerebral blood flow (CBF) monitoring. Acta Neurochir 145:1111-1115

10. Keller E, Wietasch G, Ringleb P, Scholz M, Schwarz S, Stingele R, Schwab S, Hanley D, Hacke W (2000) Bedside monitoring of cerebral blood flow (CBF) in patients with acute hemispheric stroke. Crit Care Med 28:511-516 
11. Kety SS, Schmidt CF (1948) The nitrous oxide method for the quantitative determination of cerebral blood flow in man: theory, procedure and normal values. J Clin Invest 27:476483

12. Leenders KL, Perani D, Lammertsma AA, Heather JD, Buckingham P, Healy MJR, Gibbs JM, Wise RJS, Hatazawa J, herold S, Beaney RP, Brooks DJ, Spinks T, Rhodes C, Frackowiak RSJ, Jones T (1990) Cerebral blood flow, blood volume and oxygen utilization. Brain 113:27-47

13. McCormick PW, Stewart M, Goetting MG, Dujovny M (1991) Noninvasive cerebral optical spectroscopy for monitoring cerebral oxygen delivery and hemodynamics.. Crit Care Med 19:89-97

14. Obrist WD, Thompson HK, Wang HS, Wilkinson WE (1975) Regional cerebral blood flow estimated by 133Xe inhalation. Stroke 6:245-256

15. Roberts I, Fallon P, Kirkham FJ, Loyd-Thomas A, Cooper C, Maynard R, Elliot M, Edwards AD (1993) Estimation of cerebral blood flow with near infrared spectroscopy and indocyanine green. Lancet 342:1425

16. Rossi S, Buzzi F, Paparella A, Mainini P, Stocchetti N (1998) Complications and safety associated with ICP monitoring: a study of 542 patients. Acta Neurochir Suppl 71:91-93

17. Schwarz G, Litscher G, Kleinert R, Jobstmann R (1996) Cerebral oximetry in dead subjects. J Neurosurg Anetshesiol 8:189-193

18. Sourbron S, Ingrisch M, Siefert A, Reiser M, Hermann K (2009) Quantification of cerebral blood flow, cerebral blood volume, and blood-brain-barrier leakage with DCEMRI. Magn Reson Med 62:205-217

19. Vajloczy P, Horn P, Thome C, Munch E, Schmiedek P (2003) Regional cerebral blood flow monitoring in the diagnosis of delayed ischemia following aneurysmal subarachnoid hemorrhage. J Neurosurg 98:1227-1234 